\title{
e-interview
}

columns

\section{Sheilagh Hodgins}

Sheilagh Hodgins is Professor and Head, Department of Forensic Mental Health Science, Institute of Psychiatry, King's College, University of London. She trained at McGill University, Canada. Her areas of special interest are the links between the major mental disorders and criminality and violent behaviour.

If you were not a psychologist, what would you do?

I do not see myself as a psychologist but rather as a scientist. I have never really related to a professional discipline, but always thought that knowledge from many disciplines was necessary to understand and to solve most problems that interes me. Had I not become what I am, I probably would have studied some branch of neuroscience. What I dream of is being a dancer!

\section{Do you feel stigmatised by your profession?}

No, I feel very privileged. I am allowed to think and to study and I get paid for it! I think, however, that this privilege brings with it the burden of using knowledge to solve problems. I don't mean to be pompous, but I honestly do believe that scientists can have an enormous impact on society and are the only real hope for improving the quality of life of billions of people.

Who was your most influential trainer, and why?

Professor Donald Hebb, an eminent neuroscientist, was most important. He helped me appreciate that in order to understand people, it was necessary to focus on the complex interactions between biological and non-biological factors.

\section{What job gave you the most useful} training experience?

There were actually three. The first was working in the back ward of a large psychiatric hospital with patients with chronic schizophrenia. The second most useful training experience was going to the homes of families with pre-school aged boys with severe conduct disorder and/or attentiondeficit hyperactivity disorder. The third most useful training experience was working in a clinic for sexual dysfunctions. There I saw that problems that had historically been viewed as enduring symptoms of personality disturbance could be successfully resolved with minimal interventions, sometimes just by reading the right book. This led me to ask why professionals sometimes make things more complicated than they are?

\section{Which book/text has influenced you} most?

I love to read, but honestly I cannot think of 'one' book that has influenced me more

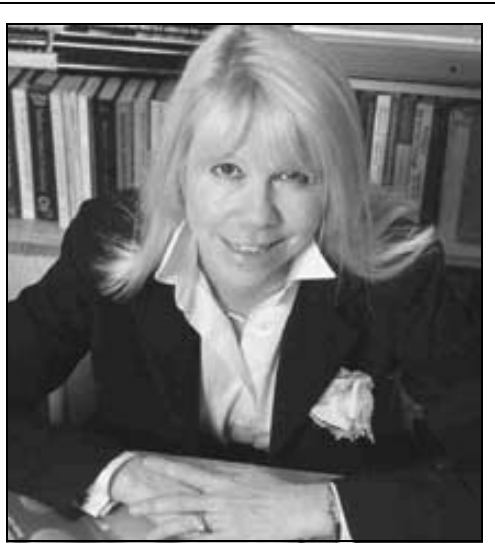

than others. One that I often re-read is written by students of Karl von Linné who he sent to visit North America. Linné had chosen these particular students to make the long voyage from Sweden to North America because of their keen observational skills. They visited Niagara Falls and Montreal in 1770, two places I know well (I grew up about 7 km from Niagara Falls, Canada, and I lived in Montreal from the age of 18). Linné's students wrote vivid descriptions of the people they met, their habits and culture, as well as the countryside and of course all the plants. I greatly admire their skill in observing behaviours. Each time I reread this book I think about the profound changes and progress that have occurred since that time, the fact that people's lives are much easier physically, but much more difficult in other ways. This suggests that the types of individuals who may have thrived in the late 18th century are not the types who will likely thrive now. Furthermore, re-reading this book reminds me of the extent to which these changes have involved destruction of a beautiful land.

What part of your work gives you the most satisfaction?

The most satisfaction comes from finding answers to a question or being able to pull together disparate and confusing findings into one logical explanation. Equally satisfying is convincing decision-makers to use research findings to modify mental health policies and practices

\section{What do you least enjoy?}

Filling out forms and writing documents for bureaucrats that have no impact on anything except the numbers of trees that are left on the earth.

\section{What conflict of interest do you} encounter most often?

The most common ones that I see these days are disagreements among scientists about ownership of data sets, individuals reviewing grant applications and manuscripts written by persons with whom they have some kind of conflict or emotional relationship, and issues about funding from pharmaceutical companies and other non-academic bodies. I also observe individuals struggling to decide how to present findings from studies. Such situations do not involve misrepresenting or changing data, but rather a realistic appreciation that the way in which the data are presented will determine, to a large extent, the reactions they provoke, and in some cases, decisions about healthcare policy or practices, or scientific funding.

Do you think psychiatry is brainless or mindless?

Absolutely not! However, the discipline does have to promote science, both as a necessary expertise for trainees and as a basis for change and progress.

What are the main ethical problems that psychiatrists will face in the future?

Control. Thinking the professional knows best what the patient needs but respecting the patients' right to make decisions about themselves and promoting their autonomy.

How would you improve clinical psychiatric training?

I don't think that all clinicians need to be scientists, but they all need to learn about science. Clinicians have to learn how to use new knowledge to modify their practice and accept that change in practice is the norm and a mark of progress. To accomplish this they have to learn to critically read and understand reports of scientific studies, and accept that it is part of their professional responsibility to update their knowledge of the types of patients they treat and the effectiveness of various interventions for these types of patients.

What single change to mental health legislation would you like to see? An acknowledgement that mental health problems, including behavioural disorders, are as deserving of money and services as somatic problems. A health system oriented to prevention, rather than treatment.

What is the future for psychotherapy in psychiatry training and practice?

For the problems that I study, the major mental disorders and antisocial behaviour, and antisocial behaviour that is stable from childhood across the life span, traditional individual psychodynamically oriented psychotherapy has been shown to be ineffective. Cognitive-behavioural and behaviour therapies show more promise, but much remains to be learned about specific interventions for different problems and different types of patients, about how to integrate such treatments into current services, and the time when such interventions achieve maximum effectiveness.

Dominic Fannon 